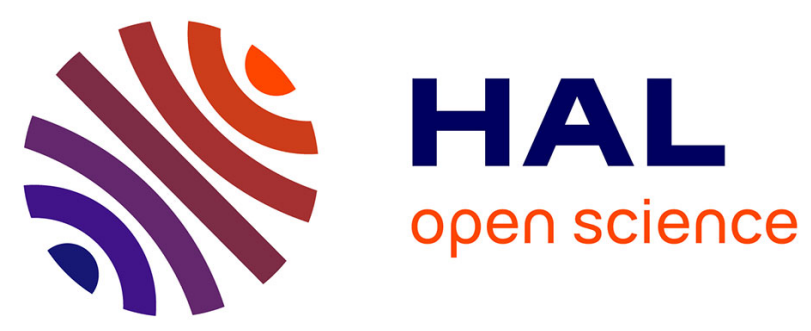

\title{
Self-similar regimes of turbulence in weakly coupled plasmas under compression
}

\author{
Giovanni Viciconte, Benoît-Joseph Gréa, Fabien S. Godeferd
}

\section{To cite this version:}

Giovanni Viciconte, Benoît-Joseph Gréa, Fabien S. Godeferd. Self-similar regimes of turbulence in weakly coupled plasmas under compression. Physical Review E , 2018, 97, pp.023201. 10.1103/PhysRevE.97.023201 . hal-02084835

\section{HAL Id: hal-02084835 \\ https://hal.science/hal-02084835}

Submitted on 21 Feb 2020

HAL is a multi-disciplinary open access archive for the deposit and dissemination of scientific research documents, whether they are published or not. The documents may come from teaching and research institutions in France or abroad, or from public or private research centers.
L'archive ouverte pluridisciplinaire HAL, est destinée au dépôt et à la diffusion de documents scientifiques de niveau recherche, publiés ou non, émanant des établissements d'enseignement et de recherche français ou étrangers, des laboratoires publics ou privés. 


\title{
Self-similar regimes of turbulence in weakly coupled plasmas under compression
}

\author{
Giovanni Viciconte \\ CEA, DAM, DIF, F-91297 Arpajon, France and LMFA UMR5509, CNRS, Université de Lyon, École Centrale de Lyon, \\ Université Claude Bernard Lyon 1, INSA Lyon, Écully, France \\ Benoît-Joseph Gréa \\ CEA, DAM, DIF, Arpajon F-91297, France
}

Fabien S. Godeferd

LMFA UMR5509, CNRS, Université de Lyon, École Centrale de Lyon, Université Claude Bernard Lyon 1, INSA Lyon, Écully, France

(Received 4 September 2017; revised manuscript received 5 January 2018; published 8 February 2018)

\begin{abstract}
Turbulence in weakly coupled plasmas under compression can experience a sudden dissipation of kinetic energy due to the abrupt growth of the viscosity coefficient governed by the temperature increase. We investigate in detail this phenomenon by considering a turbulent velocity field obeying the incompressible Navier-Stokes equations with a source term resulting from the mean velocity. The system can be simplified by a nonlinear change of variable, and then solved using both highly resolved direct numerical simulations and a spectral model based on the eddy-damped quasinormal Markovian closure. The model allows us to explore a wide range of initial Reynolds and compression numbers, beyond the reach of simulations, and thus permits us to evidence the presence of a nonlinear cascade phase. We find self-similarity of intermediate regimes as well as of the final decay of turbulence, and we demonstrate the importance of initial distribution of energy at large scales. This effect can explain the global sensitivity of the flow dynamics to initial conditions, which we also illustrate with simulations of compressed homogeneous isotropic turbulence and of imploding spherical turbulent layers relevant to inertial confinement fusion.
\end{abstract}

DOI: 10.1103/PhysRevE.97.023201

\section{INTRODUCTION}

In inertial confinement fusion (ICF), it is essential to predict how the light elements composing the fuel, typically deuterium-tritium, evolve during the target compression. In order to achieve ignition, the fuel has to be brought to very high temperature corresponding to the plasma state. The turbulent mixing generated by hydrodynamics instabilities taking place at the fuel-ablator interface may however cool the core and consequently deteriorate the capsule yield. Recently, different simulations by [1] showed that the growth of the viscosity coefficient, as the deuterium-tritium plasma heats up to the kinetic regime [2], dissipates the turbulent kinetic energy. It also inhibits the growth of Rayleigh-Taylor or Kelvin-Helmholtz instabilities [3], impacts the mean radial flow [4], and possibly influences the turbulent mixing in the central hot spot, where fusion processes set in [5]. The suddenness of this effect can substantially participate in a local temperature increase by rapidly converting the kinetic energy into an internal one [6]. Meanwhile, the initial conditions and particularly the large scale asymmetries of the capsule, due for instance to the support tent or to the fill tube [7], play a determining role by having a strong imprint on the turbulent mixing. Still, how the balance between the stirring processes and the microscopic dissipation is influenced by initial conditions remains unclear in ICF imploding capsules.

In this context, we propose to study the importance of initial conditions by considering the simplified case of homogeneous isotropic turbulence (HIT) under compression, previously introduced by [6]. In this configuration, the plasma turbulence dynamics is expressed by the Navier-Stokes equations with a viscosity coefficient dependent on the mean temperature. In addition, the turbulence production is impulsed solely by the compression terms coming from the mean velocity field while other production sources such as shear or density gradient are neglected. Despite being crude, this framework has already proven an interesting starting point to explore important mechanisms at work during the compression, namely turbulence production and dissipation but also nonlinear transfer. In order to extend our analysis to a less academic geometry and to an inhomogeneous flow, we also consider in this paper a spherical turbulent layer with parameters relevant to ICF, as a paradigm of the hot spot contamination by turbulence.

The isotropic compression of turbulence obeying the Navier-Stokes equations is a classical problem in fluid dynamics. Assuming incompressible turbulent fluctuations and exploiting the similarity properties of the equations, the forcing compression term can be eliminated by a coordinate transformation [8]. The turbulence expressed in the new moving frame decays at a rate which may be influenced by the time-varying viscosity coefficient appearing due to the coordinate transformation. These properties have been exploited by $[9,10]$ in order to propose practical turbulence models and to evaluate the growth of turbulent kinetic energy back in the laboratory frame.

Meanwhile, it is known since the work of Batchelor [11,12] followed by [13] that the large-scale structures or big eddies play an important part in the dynamics of turbulent flows. 
This role has been identified for instance through different self-similar solutions, corresponding to the turbulent and final decays of HIT, showing a dependence on the initial distribution of energy at large scales. These solutions have been investigated in particular by spectral models based on eddy-damped quasinormal Markovian (EDQNM) closures [14,15] allowing a systematic exploration of the influence of initial conditions at very high Reynolds number [16-18]. Note that this method has been similarly generalized to other problems such as Rayleigh-Taylor and unstably stratified turbulence [19-21].

In this paper, we identify different regimes of turbulence in a plasma under compression by exploiting the similarities properties of the system and the possibility of finding selfsimilar solutions in the moving frame corresponding to the coordinate transformation. Back into the laboratory frame, this gives information about the importance of initial conditions. In order to achieve this goal, we use direct numerical simulations (DNSs) and spectral models based on classical EDQNM closures. The two methods are complementary: DNS provides the details of the turbulent fields in space, and their time evolution, while, by construction, the EDQNM model directly predicts the time-evolving turbulent spectra of twopoint correlations, and allows exhaustive parametric studies as well as the exploration of high Reynolds number regimes, due to its low computational cost.

This paper is organized as follows: first we present the basic equations used to investigate the evolution of turbulent quantities under compression, as well as the EDQNM model validated by comparison to DNS. Then, we identify the theoretical regimes and scaling laws appearing in the turbulence evolution during different phases of the compression. All these solutions are then explored at higher Reynolds number by EDQNM simulations, and illustrated in the case of an imploding spherical turbulent layer.

\section{THEORETICAL FRAMEWORK, SIMULATIONS, AND MODEL FOR A TURBULENT PLASMA UNDER COMPRESSION}

In this part, we introduce the theoretical framework describing a turbulent plasma under compression, and we explain how DNS and the EDQNM model can be efficiently used to solve the resulting system of equations.

\section{A. Basic equations}

We first recall the basic equations of turbulence subjected to an isotropic compression and previously detailed in $[6,10]$. In these works and also the present one, the problem is restricted to a hydrodynamic framework. For plasmas originating from large ICF capsules, such as described in [1], it is usually considered as a good starting point to study the problem. Indeed, the typical Knudsen numbers evaluated in this case are consistent with the continuum hypothesis. Also, the kinetic energy density much greater than the magnetic one, see the typical values of self-induced magnetic field in [22], allows us to neglect magnetohydrodynamics effects.

Therefore, our analysis begins with the Navier-Stokes and mass conservation equations for a compressible fluid in a Cartesian stationary reference frame $\mathbf{x}$. We use the classical
Reynolds decomposition based on the ensemble average. All quantities are decomposed into a superposition of mean and fluctuating (or zero mean) parts. In order to further simplify the problem, we will require that fluctuating turbulent quantities are homogeneous. This imposes a mean velocity $\mathbf{U}(\mathbf{x}, t)$ on the form (see [23]):

$$
\mathbf{U}(\mathbf{x}, t)=-\mathbf{x} \mathcal{S}(t),
$$

introducing the compression time rate $\mathcal{S}(t)$. Considering a particle moving with the mean velocity $\mathbf{U}$, its distance to the center decreases by a factor $L(t)=\exp \left(-\int_{0}^{t} \mathcal{S}(s) d s\right)$, referred to as the compression parameter. If we inject this definition into the mean-field equation, we find that for $L(t)$, a linear function of time, the mean pressure gradient is zero. This is the same choice of [6]: $L(t)=1-\mathcal{S}_{0} t$ for $t<1-\mathcal{S}_{0}$ with $\mathcal{S}_{0}=$ $\mathcal{S}(0)$, which also amounts to $\mathcal{S}(t)=\mathcal{S}_{0} /\left(1-\mathcal{S}_{0} t\right)$. Considering this particular compression rate allows us to discard density fluctuation effects or classical Rayleigh-Taylor instabilities usually appearing during the compression when mean pressure gradients are present.

From the given form of $\mathbf{U}$ and the hypothesis that the mean density $\rho$ is uniform, the mass conservation equation shows that $\rho(t)$ evolves as $\rho(t)=\rho_{0} L(t)^{-3}$ (from now on, the initial value of a quantity is denoted by the suffix ${ }_{0}$ ).

We now turn our attention to the fluctuating velocity, $\mathbf{u}(\mathbf{x}, t)$, whose equations are given by

$$
\begin{aligned}
\partial_{t} \mathbf{u}+\mathbf{u} \cdot \nabla \mathbf{u}-\mathcal{S}(t) \mathbf{x} \cdot \nabla \mathbf{u}-\mathcal{S}(t) \mathbf{u} & =-\nabla p+\frac{\mu(t)}{\rho(t)} \nabla^{2} \mathbf{u} \\
\nabla \cdot \mathbf{u} & =0
\end{aligned}
$$

where $p$ is the fluctuating reduced pressure. Note that the fluctuating velocity is assumed divergence free (unlike the mean velocity part $\mathbf{U}$ ). It is indeed possible to consider an incompressible turbulent velocity field if the compression and turbulent time scales are larger to the characteristic time corresponding to pressure effects (low Mach approximation). This is extensively detailed in [24] for instance.

Equation (2a) contains an inhomogeneous term, $\mathcal{S}(t) \mathbf{x} \cdot \nabla \mathbf{u}$, coming from the advection of turbulence by the mean field. In order to remove this inhomogeneous dependence, we rescale the space coordinates as

$$
\tilde{\mathbf{x}}=\frac{\mathbf{x}}{L(t)}
$$

This is a classical transformation from Eulerian to Lagrangian coordinates [25]; this choice has proven to be an invariant rescaling of the Navier-Stokes equation [8]. The new acceleration term that comes from this transformation exactly balances the inhomogeneous term in Eq. (2a). This is one advantage of the method, which still remains limited to linear dependence between $\mathbf{U}$ and $\mathbf{x}$ [8] (i.e., the case of homogeneous isotropic compression). After transformation, we have

$$
\partial_{t} \mathbf{u}+\frac{1}{L} \mathbf{u} \cdot \tilde{\nabla} \mathbf{u}-\mathcal{S}(t) \mathbf{u}=-L^{2} \tilde{\nabla} p+\frac{\mu(t)}{\rho(t) L^{2}} \tilde{\nabla}^{2} \mathbf{u} .
$$


The forcing term that is still present can be eliminated using a rescaling of the variables [10],

$$
\begin{aligned}
\tilde{t} & =\int_{0}^{t} L^{-2}(s) d s \quad \tilde{\mathbf{u}}(\tilde{\mathbf{x}}, \tilde{t})=L(t) \mathbf{u}(\mathbf{x}, t), \\
\tilde{p}(\tilde{\mathbf{x}}, \tilde{t}) & =L^{5}(t) p(\mathbf{x}, t),
\end{aligned}
$$

such that quantities expressed in the "moving" frame are now identified by the symbol $\tilde{*}$.

The dynamic viscosity coefficient $\mu$ depends on the temperature $T$ as $\sim T^{5 / 2}$ for a plasma in the kinetic regime [2]. Following [6], we assume adiabatic compression so that the time evolution of viscosity is $\mu(t)=\mu_{0} L(t)^{-5}$ for a monoatomic ideal gas. The system (2) becomes

$$
\begin{aligned}
\partial_{\tilde{t}} \tilde{\mathbf{u}}+\tilde{\mathbf{u}} \cdot \tilde{\nabla} \tilde{\mathbf{u}} & =-\tilde{\nabla} \tilde{p}+v_{0} \tilde{L}^{-2} \tilde{\nabla}^{2} \tilde{\mathbf{u}}, \\
\tilde{\nabla} \cdot \tilde{\mathbf{u}} & =0,
\end{aligned}
$$

where the initial kinematic viscosity is $v_{0}=\mu_{0} / \rho_{0}$. Equations (6a) and (6b) correspond to the Navier-Stokes equations for $\tilde{\mathbf{u}}(\tilde{\mathbf{x}}, \tilde{t})$ with a time-increasing kinematic viscosity coefficient $\tilde{v}(\tilde{t})=v_{0} \tilde{L}^{-2}(\tilde{t})$, where $\tilde{L}(\tilde{t})=L(t)$. In this moving frame, an initially homogeneous turbulence remains so during the compression. This new frame is significantly advantageous, since it converts the problem of compressed turbulence into that of HIT decay with time-varying viscosity. And since the velocities in fixed and moving frame are linked by a factor that depends only on time, the velocity fluctuation in the fixed frame is homogeneous. This confirms the initial hypotheses on the homogeneity of the fluctuating field.

Two nondimensional numbers permit us to fully characterize the flow regime: the Reynolds number Re and the compression number $\mathrm{Cp}$, defined at initial time as

$$
\operatorname{Re}_{0}=\frac{u_{0} \ell_{0}}{v_{0}} \text { and } \mathrm{Cp}_{0}=\frac{u_{0}}{\ell_{0} \mathcal{S}_{0}},
$$

where $\ell_{0}$ and $u_{0}$ are characteristic length and velocity scales of the initial turbulent flow. Re measures the ratio between the turbulent to the physical viscosity, and $\mathrm{Cp}$ the ratio between the turbulent frequency to the compression rate. It informs about the initial conditions of the system and helps to determine which physical phenomenon can become dominant during the different phases of compression.

\section{B. Direct numerical simulations and the EDQNM model for compressed turbulence}

In order to quantify and analyze the different phenomena involved in the flow dynamics, and in particular to illustrate the competition between turbulence production and viscous dissipation, we present results from well resolved DNSs along with predictions by the spectral EDQNM model.

Our DNS code solves the system of Eqs. (6) associated to the coordinates in the moving frame. The system used in [6] is slightly different and has an additional source term due to a different choice of coordinate transformation. In the representation corresponding to Eqs. (6), the numerical resolution is not an issue since the viscosity coefficient keeps growing. Our code uses a classical pseudospectral numerical method in a three-dimensional $2 \pi$-periodic domain already used by [26], from which it differs only by the time-varying

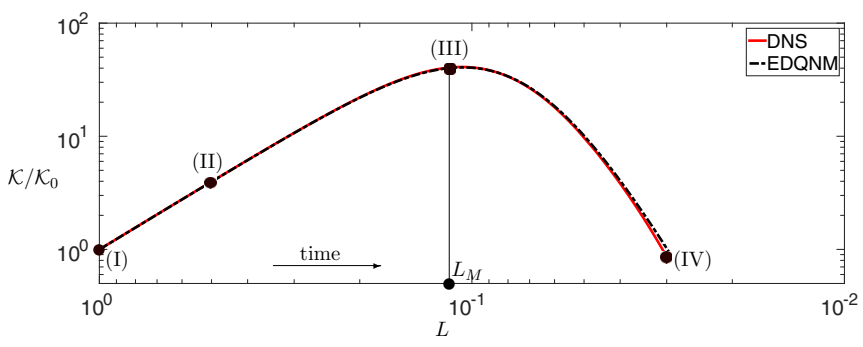

FIG. 1. Evolution of turbulent kinetic energy $\mathcal{K}$ as a function of the compression parameter $L$ for both DNSs at resolution $512^{3}$ and EDQNM simulations of a HIT compression case with $\operatorname{Re}_{0}=250$ and $\mathrm{Cp}_{0}=0.1$. The critical compression parameter $L_{M}$ corresponds to the kinetic-energy maximum before the beginning of the viscous phase.

viscosity which is treated by an explicit third-order RungeKutta scheme. A validation of this method has been presented in [27], showing in particular its ability to reproduce the simulation results of [6].

We also use a spectral model adapted from the isotropic EDQNM closure [15], and extended to treat turbulence under compression (see details in [27]). The EDQNM model applied to Eqs. (6) allows us to compute the evolution of the turbulent kinetic-energy spectra $\tilde{E}(\tilde{k})$ as a function of the wave number $\tilde{k}$ in the moving frame. Both are then transformed back to $k$ and $E(k)$ in the initial fixed frame. The EDQNM model proposed here is close to the original homogeneous isotropic versions presented in $[14,15]$ except for the choice of the eddy damping term which takes into account the time-varying viscosity, modifying the Markovianization process. The spectral EDQNM model also reproduces adequately the results of [6] and of other moderate Reynolds number DNS.

We illustrate this in Figs. 1 an 2, with results obtained using an initial kinetic-energy spectrum of the Batchelor form $E_{0}(k) \sim k^{4} \exp \left[-2\left(k / k_{\text {peak }}\right)^{2}\right]$, where $k_{\text {peak }}$ corresponds to the maximum of $E_{0}$. The initial Reynolds number is $\operatorname{Re}_{0}=250$ and the compression number $\mathrm{Cp}_{0}=0.1$, based on $\ell_{0}=1 / k_{\text {peak }}$ and $u_{0}=\mathcal{K}^{1 / 2}$. Turbulence is therefore relatively weak in this example, while the compression is rapid compared to the turbulent time scale.

In Fig. 1, we show the evolution of turbulent kinetic energy $\mathcal{K}=\int_{0}^{+\infty} E(k) d k$ as a function of the compression parameter $L(t)$. We observe a very good agreement between simulations of $512^{3}$ grid-point DNSs and the EDQNM model during all the phases of the kinetic-energy evolution. Moreover, the spectral distribution of energy at four instants, plotted in Fig. 2 (bottom row), shows a very good agreement between DNSs and the EDQNM model at all scales. This supports the relevance of the closure as a model for compressed turbulence.

The case presented in Figs. 1 and 2 is typical of the regime extensively discussed in [6]. At the beginning $[L(t)$ from 1 to $O\left(10^{-1}\right)$ ], the dynamics of the flow is dominated by the compression effects leading to the increase of kinetic energy. Progressively, viscosity grows and dissipates energy in the small scales and eventually at larger scales, as indicated by the spectra. This counterbalances the turbulence production mechanisms and finally triggers the sudden viscous dissipation effect. These mechanisms are qualitatively observed in Fig. 2 (top row) which shows the spatial distribution of kinetic energy 


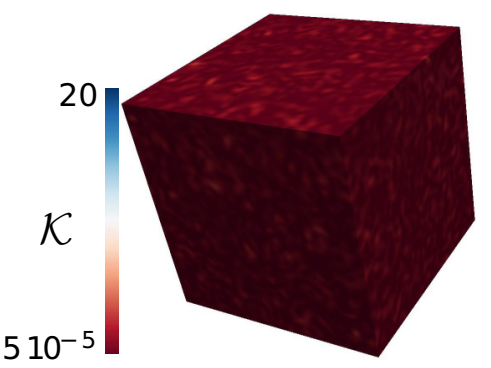

(I)

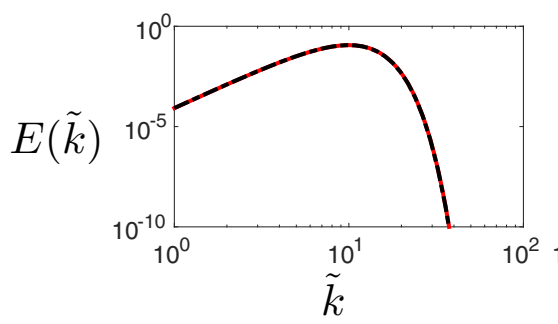

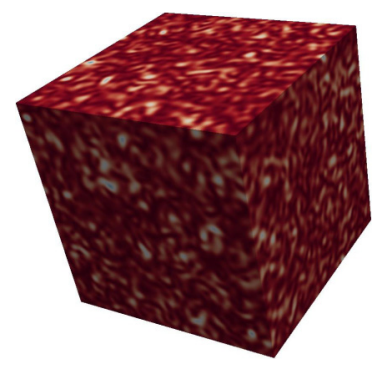

(II)

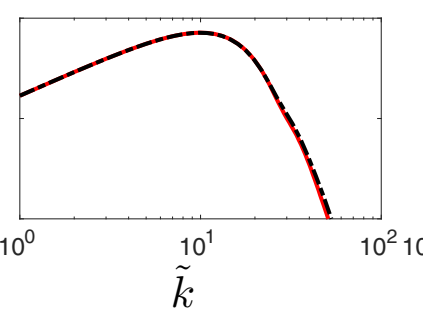

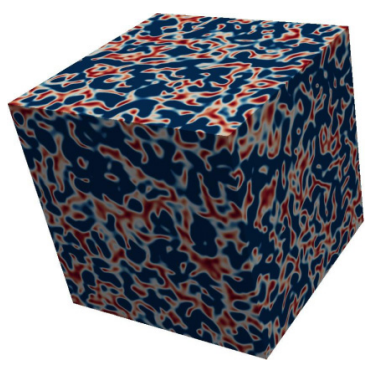

(III)

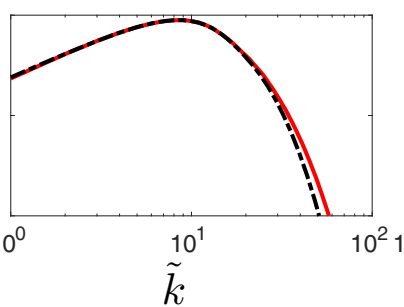

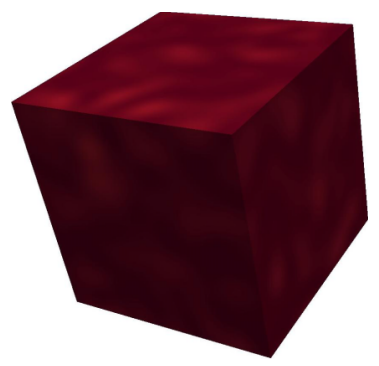

(IV)

FIG. 2. Top row: distribution of turbulent kinetic energy $\mathcal{K}$ in the DNS at four instants of the evolution, indicated in the kinetic energy curve of Fig. 1. The scaling of the box corresponds to the moving frame of reference. Bottom row: Associated energy spectra $E(\tilde{k})$ for both DNS and EDQNM simulations, at the same instants.

in the DNS domain at the same four instants as the presented spectra. One particularly observes the intensification of kinetic energy, especially at instant (III) corresponding to the peak of kinetic energy in Fig. 1, and the strong reduction of energy levels at instant (IV), along with a smoothing of the field due to the damping of small-scale fluctuations.

The critical value $L_{M}$ of the compression ratio corresponds to the maximum of kinetic energy in Fig. 1. It indicates how much the turbulence can be compressed before the appearance of the sudden viscous dissipation effect. We have therefore computed the evolution of $L_{M}$ with the two relevant nondimensional parameters, Reynolds and compression numbers, the initial spectrum remaining of the Batchelor form. The corresponding map of $L_{M}$ in the (Re, Cp) coordinates is shown in Fig. 3.

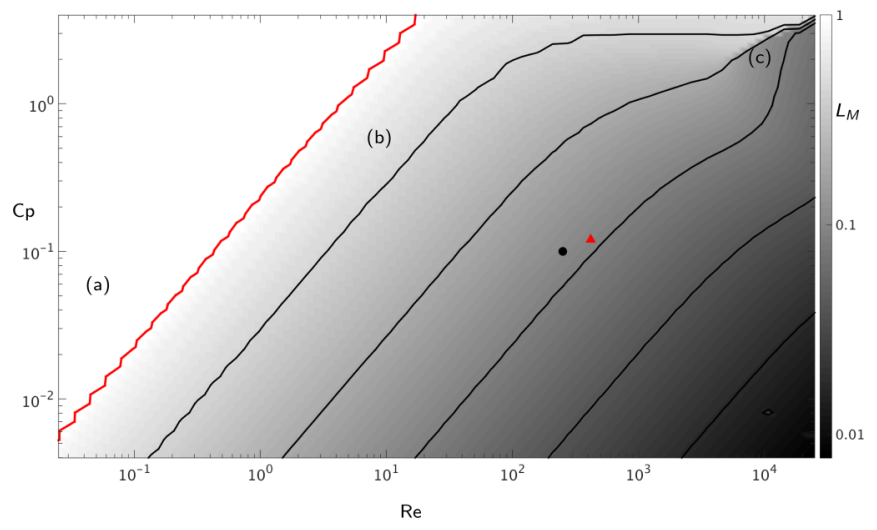

FIG. 3. Isocontour map of the critical compression parameter $L_{M}$ corresponding to maximum of kinetic energy, as a function of the initial Reynolds number $\mathrm{Re}_{0}$ and the compression number $\mathrm{Cp}_{0}$. Results from EDQNM simulations. The black circle corresponds to the parameters used in Figs. 1 and 2, and the red triangle to that of Fig. 8. The red line is the contour line at $L_{M}=1$.
This parametric study was permitted by the EDQNM model, which allows us to explore six decades of initial Reynolds numbers $\operatorname{Re}_{0}$ and three decades of initial compression number $\mathrm{Cp}_{0}$. It required as many as 10000 EDQNM simulations, which would not be possible using DNSs due to its high computational cost.

As expected, Fig. 3 shows two general trends. First, when the initial Reynolds number increases, $L_{\mathrm{M}}$ decreases since the viscosity coefficient needs to grow enough in order to impact the main energetic scales. Second, for decreasing values of the compression number $\mathrm{Cp}_{0}$, the critical compression parameter $L_{\mathrm{M}}$ also becomes smaller since turbulent production is stronger and needs more time before being balanced by dissipation.

Upon closer inspection, the isolines of $L_{M}$ in Fig. 3 permit us to identify two additional kinds of dynamics different from that presented in Fig. 1, wherein turbulent production dominance is followed by that of viscous dissipation as $L$ decreases. The first additional regime occurs at small initial $\operatorname{Re}_{0}$ and large $\mathrm{Cp}_{0}$, and is also observed in [6]: dissipation immediately prevails from the very beginning of the compression phase, and yields a decay of turbulent kinetic energy, and a narrow energy spectrum limited to large scales, as in Fig. 4(a).

The second additional regime appears at large values of $\mathrm{Re}_{0}$ and $\mathrm{Cp}_{0}$, and corresponds to the presence of important nonlinear energy transfers. In this new regime, two values of the compression parameter, corresponding to two kineticenergy maxima, may exist. This generates a discontinuity observed in Fig. 3 [top-right corner, at large Re and Cp, where label (c) appears] which can be explained by considering the following concurrent phenomena. First, kinetic energy grows as $L^{-2}$, which means it remains constant in the moving frame. In the mean time, energy is transferred to small scales by nonlinear effects and is then suddenly dissipated when reaching the viscous scales, in the classical mechanism of enstrophy blowup well known in HIT decay. This sudden energy loss can counterbalance the growth of kinetic energy if the Reynolds 

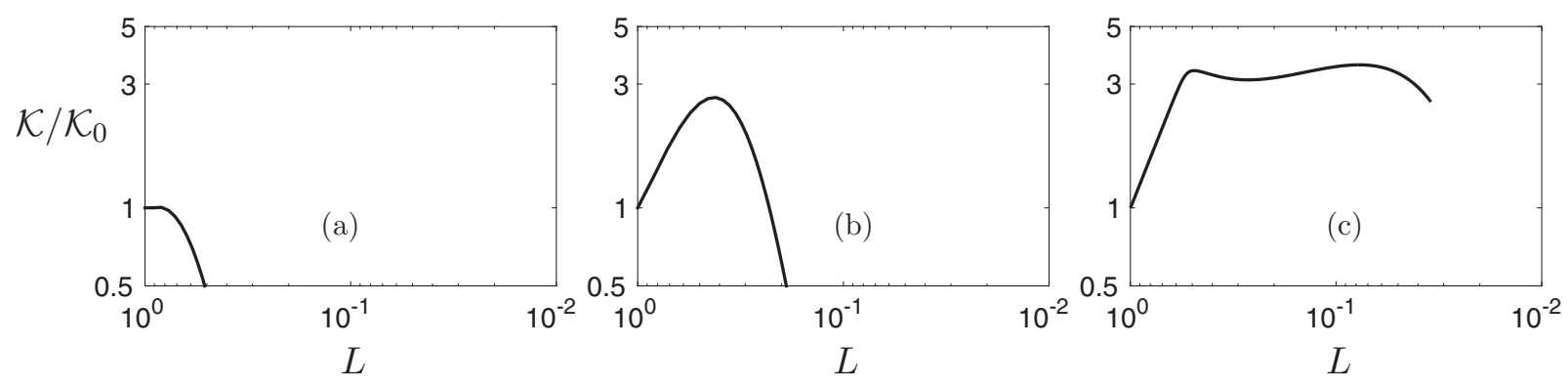

FIG. 4. Evolution of kinetic energy $\mathcal{K}$ normalized by its initial value as a function of the compression parameter $L$ for the three typical cases indicated on the parametric map of Fig. 3. The spectrum in region (a) illustrates the immediate decay regime, that of region (b) the intermediate regime, and region (c) shows the cascade regime.

number is not large enough, leading to a first maximum of $\mathcal{K}(L)$. Meanwhile, large scales keep gaining energy due to the compression until dissipated by the sudden growth of the viscosity coefficient, resulting in the second maximum shown in the spectrum in Fig. 4(c).

In summary, we have explored the influence of initial nondimensional parameters on the dynamics of turbulent plasma under compression by combining results of DNS and EDQNM simulations. This has permitted us to identify the importance of the various physical mechanisms involved during the compression. In particular, we have shown that nonlinear energy transfer can have a decisive role at high Reynolds number. In the coming section, we propose theoretical arguments in support of these conclusions.

\section{SELF-SIMILAR SOLUTIONS IN PLASMAS UNDER COMPRESSION}

The main goal of this part is to derive the different selfsimilar solutions for turbulent plasma under compression. These solutions are first expressed in the moving frame, and then transformed back in the laboratory frame using Eq. (5). As already stated, we stress that this derivation is limited to a restricted scenario, where a part of the phenomena typically involved are not considered. Nevertheless we believe that this simplified analysis can contribute to the understanding of the different mechanism involved in the evolution of turbulence in a plasma under compression. Section III A is dedicated to the theoretical analysis, and in Sec. IIIB we show the actual existence of these regimes using DNS and EDQNM simulations.

\section{A. Self-similar scaling}

Three self-similar solutions are found, corresponding to the rapid compression regime, the cascade regime, and the viscous regime. They are listed hereafter, and for convenience the corresponding scaling laws are gathered in Table I.

\section{Rapid compression regime}

The rapid compression (RC) regime, historically referred to as rapid distortion theory-called RDT in [9], although unlike in sheared turbulence, wave numbers are not distortedis the simplest self-similar solution which can be obtained. Assuming that the turbulent time scale is much larger than the compression time, such that $\mathrm{Cp} \ll 1$, turbulence can be considered frozen in the moving frame, leading to the following energy spectrum:

$$
\tilde{E}(\tilde{k}, \tilde{t})=E_{0}(\tilde{k}), \quad \text { or } \quad E(k, t)=E_{0}[L(t) k] \times L(t)^{-1},
$$

whence the evolution of kinetic energy and of the integral length scale $\ell_{I}$,

$$
\begin{aligned}
& \mathcal{K}(t)=\int_{0}^{+\infty} E(k, t) d k \sim L^{-2}(t), \\
& \ell_{I}(t)=\frac{3 \pi}{4} \frac{\int_{0}^{+\infty} k^{-1} E(k, t) d k}{\int_{0}^{+\infty} E(k, t) d k} \sim L(t) .
\end{aligned}
$$

This self-similarity of spectrum $\tilde{E}(\tilde{k}, \tilde{t})$ is of course only strictly valid for wave numbers unaffected by viscosity. It is clear that the RC regime can only last for a limited duration, due to the $L^{-2}$ growth of the viscosity and to the nonlinear transfer in the moving frame. Note also that $\mathcal{K} \sim L^{-2}$ does not necessary imply a RC phase. For instance, redistribution of energy by nonlinear transfers can modify the shape of spectra and the value of integral length scale $\tilde{\ell}_{I}$, while still conserving the total kinetic energy.

\section{Cascade regime}

The decay of HIT is extensively discussed in most turbulence books, as for instance in [12,14,23]. According to these monographs, the decay rate of energy depends on the cascading process governed by large scales of turbulence, but not on the value of the viscosity coefficient. For compressed turbulence, although the viscosity coefficient varies in the moving frame, one can expect similarly that the dynamics at large Reynolds number is driven only by the turbulent energy flux from

TABLE I. Scaling laws corresponding to the different self-similar regimes for the different turbulent quantities and nondimensional numbers. The coefficients $n_{c}$ and $n_{v}$ are given by Eqs. (10) and (12) as a function of the infrared spectral slope $s$.

\begin{tabular}{llll}
\hline \hline & RC regime & Cascade regime & Viscous regime \\
\hline $\mathcal{K}$ & $\sim L^{-2}$ & $\sim L^{n_{c}-2}$ & $\sim L^{n_{v}-2}$ \\
$\ell_{I}$ & $\sim L$ & $\sim L^{n_{c} / 2}$ & $\sim L^{-1 / 2}$ \\
$\operatorname{Re}$ & $\sim L^{2}$ & $\sim L^{n_{c}+1}$ & $\sim L^{\left(n_{v}+1\right) / 2}$ \\
$\mathrm{Cp}$ & $\sim L^{-1}$ & $\sim L^{0}$ & $\sim L^{\left(n_{v} / 2+1\right) / 2}$ \\
\hline \hline
\end{tabular}


large to small scales. Considering a self-similar evolution of kinetic energy as $\tilde{\mathcal{K}} \sim \tilde{t}^{-n_{c}}$, the integral length scale evolves as $\tilde{\ell}_{I} \sim \tilde{t}^{1-n_{c} / 2}$. Assuming the permanence of big eddies $[11,16]$, which can be expressed by $\lim _{\tilde{k} \rightarrow 0} \tilde{E}(\tilde{k}, \tilde{t})=\tilde{k}^{s}$ ( $s$ being the infrared spectral slope), we deduce from self-similarity

$$
n_{c}=\frac{2(s+1)}{s-3} \text {. }
$$

This provides the classical decay exponent generally encountered in turbulence: $n_{c}=10 / 7 \simeq 1.43$ for the Batchelor spectrum $(s=4)$ and $n_{c}=6 / 5=1.2$ for the Saffman spectrum $(s=2)$. Note that the permanence of big eddies is relatively well observed for $s \leqslant 3$ while not completely true for $s=4$ due to backscatter effects transferring energy from small to large scales.

Returning to the laboratory frame using Eq. (5) and the expression for $L(t)$, this leads to the kinetic-energy evolution as $L \rightarrow 0$ for the cascade regime:

$$
\mathcal{K} \sim L^{n_{c}-2} \text { and } \ell_{I}(t) \sim L^{n_{c} / 2},
$$

leading in particular to a growth of kinetic energy and decrease of integral scale as $\mathcal{K} \sim L^{-4 / 5}, \ell_{I} \sim L$ for Saffman turbulence and as $\mathcal{K} \sim L^{-4 / 7}, \ell_{I} \sim L^{2}$ for Batchelor turbulence.

\section{Viscous regime}

The last regime is closely related to the sudden viscous dissipation effect occurring in a turbulent plasma under compression, discussed in [6]. When the Reynolds number becomes small enough, the decay of HIT enters a final regime which is driven by dissipation and in which the nonlinear turbulent transfer is negligible. This phase exhibits a self-similar solution known as the final decay regime of HIT when the viscosity coefficient is constant [12]. We propose here a generalization for the time-varying viscosity case corresponding to a weakly coupled plasma.

As for the turbulent decay presented in Sec. III A2, we consider a self-similar evolution in the moving frame of the form $\tilde{\mathcal{K}} \sim \tilde{t}^{-n_{v}}$. The scaling for the integral scale can be obtained from the self-similar evolution of the turbulent time $\sim \tilde{t}$ and the viscosity coefficient $\tilde{v}(\tilde{t})$, yielding $\tilde{\ell}_{I} \sim[\tilde{v}(\tilde{t}) \tilde{t}]^{1 / 2}$. Assuming again $\lim _{\tilde{k} \rightarrow 0} \tilde{E}(\tilde{k}, \tilde{t})=\tilde{k}^{s}$ as large scales remain unaffected by viscosity, we obtain, for $\tilde{L} \rightarrow 0$,

$$
n_{v}=\frac{3}{2}(s+1) \text {. }
$$

Similarly to the cascade phase, we deduce the scaling laws for the kinetic energy and integral scale in the laboratory frame for the viscous phase,

$$
\mathcal{K} \sim L^{n_{v}-2} \text { and } \ell_{I}(t) \sim L^{-1 / 2},
$$

leading in particular to a sudden viscous dissipation of the form $\mathcal{K} \sim L^{5 / 2}$ in Saffman turbulence and $\mathcal{K} \sim L^{11 / 2}$ for Batchelor turbulence. Therefore, the viscous regime corresponding to the sudden dissipation effect clearly induces an important sensitivity to initial conditions contrary to the cascade regime for instance where the variations of the growth exponent are smaller. Notably in the viscous regime, the integral scale of turbulence grows despite the compression and is not dependent on initial conditions.
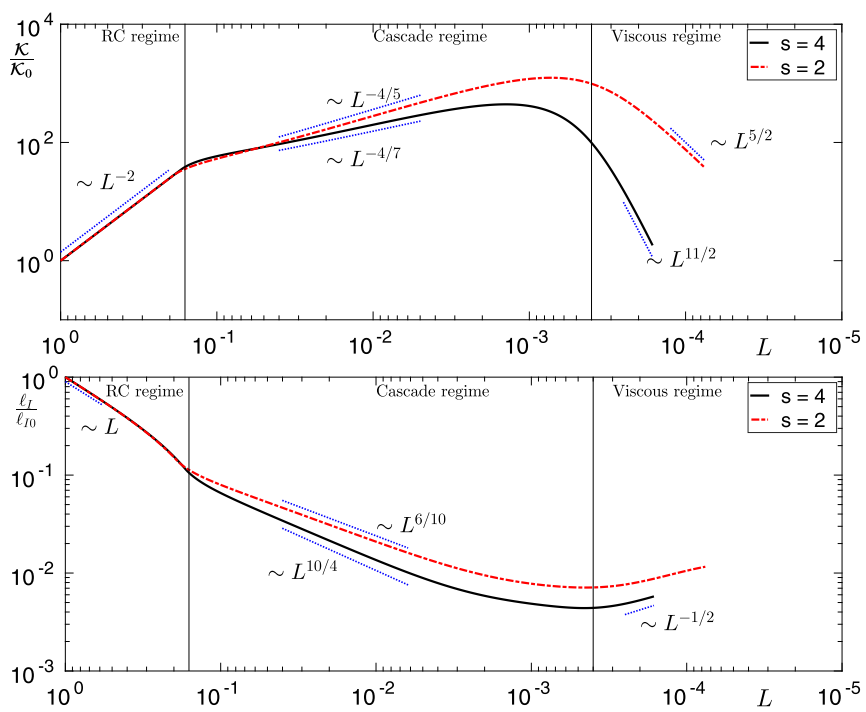

FIG. 5. Evolution of (top) the kinetic energy $\mathcal{K}$ and (bottom) the integral scale $\ell_{I}$ as a function of the compression parameter $L$ using EDQNM simulations. Solid line: Batchelor initial condition $(s=4)$. Dashed line: Saffman initial condition $(s=2)$. The scaling laws corresponding to the self-similar solutions derived in Sec. III A are also shown.

\section{B. Simulations of self-similar regimes}

We now confirm the actual appearance, in simulations, of the different self-similar solutions analytically found in the theoretical study of Sec. III A. We consider two types of initial conditions, as representative of many encountered situations: Saffman turbulence $(s=2)$ corresponding to an equipartition of energy at large scale among the different wave vectors, and Batchelor turbulence $(s=4)$ generated by backscatter effects from small scale perturbations. In Sec. III B 1, we consider the case of high Reynolds number turbulence, and for this we use the EDQNM model. In Sec. III B 2, we model the compression of a spherical turbulent layer using both DNS and EDQNM.

\section{High Reynolds number compressed turbulence with EDQNM simulations}

The Reynolds number $\operatorname{Re}=u \ell / v$ for a weakly coupled turbulent plasma under compression is expected to decrease, as assessed by the scaling laws for $u=\mathcal{K}^{1 / 2}$ and $\ell=\ell_{I}$ in either the rapid compression or the cascade or viscous regime. Thus, we use the EDQNM model to reach a very high initial Reynolds number, beyond the possibilities of DNS, in order to obtain the different self-similar solutions derived in Sec. III A.

We choose initial spectra in the von Karman type $E_{0}(k)$ $\sim k^{s} \exp \left[-s / 2\left(k / k_{\text {peak }}\right)^{2}\right]$, both for the Saffman $(s=2)$ and the Batchelor $(s=4)$ cases. We set the Reynolds number at $\operatorname{Re}_{0}=10^{7}$ and the compression number $\mathrm{Cp}_{0}=0.47$. Accordingly, this corresponds to a flow with high intensity turbulent fluctuations and to fast compression.

Figure 5 shows the evolutions of the kinetic energy $\mathcal{K}$ and of the integral length scale $\ell_{I}$, and Fig. 6 shows that of the Reynolds number Re and of the compression number $\mathrm{Cp}$. The corresponding spectra $E(k)$ at different stages of the evolution are given in Fig. 7. 

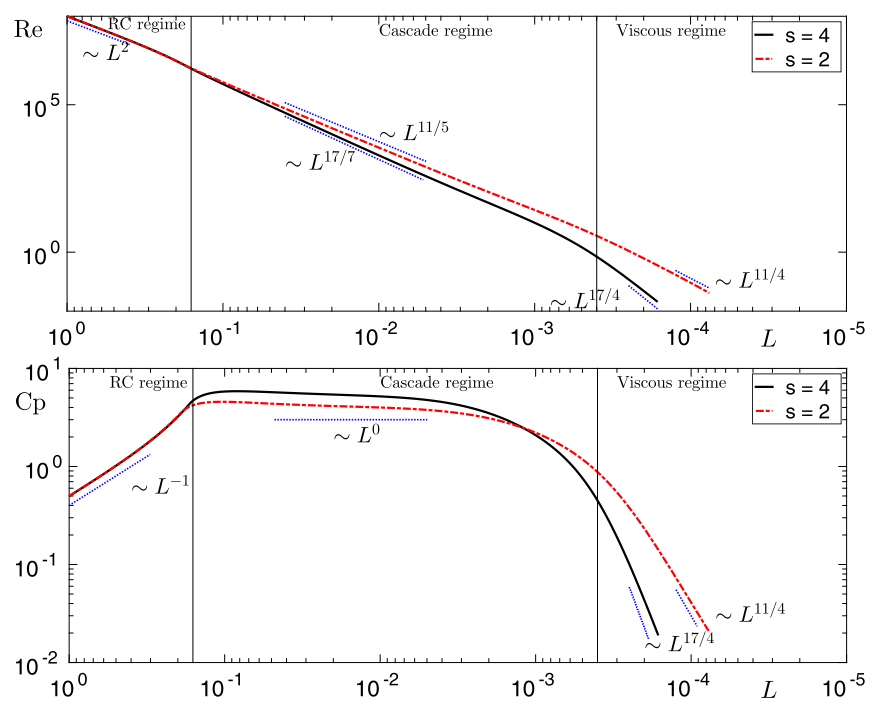

FIG. 6. Evolution of (top) the Reynolds number Re and (bottom) the compression number $\mathrm{Cp}$ as a function of the compression parameter $L$ using EDQNM simulations. Solid line: Batchelor initial condition $(s=4)$. Dashed line: Saffman initial condition $(s=2)$. The different scaling laws corresponding to the self-similar solutions derived in Sec. III A are also shown.

Figures 5 and 6 (bottom) exhibit very clearly the three different regimes that can be identified by the evolution changes in $K, \ell_{I}$, and $\mathrm{Cp}$ when $L$ decreases. The dynamical changes in the evolution of Re (Fig. 6, top) are less obvious due to transitions between similar power laws, especially the RC to cascade one, but are still present. For all curves, the self-similar scaling laws proposed in Sec. III A fit adequately the results of simulations. The succession of rapid compression, cascade, and viscous regimes is clear, and the values of $L$ at which the RC-cascade transition occurs seem to be similar for Batchelor and Saffman turbulences.

A greater sensitivity to initial conditions in the viscous regime for the turbulent kinetic-energy evolution is observed in Fig. 5, but the integral length scale increase in this regime is similar for both Saffman and Batchelor cases. We conclude that differences in the integral scale $\ell_{I}$ dynamics are only due to the cascade phase, thus demonstrating the imprint of nonlinear mechanisms.

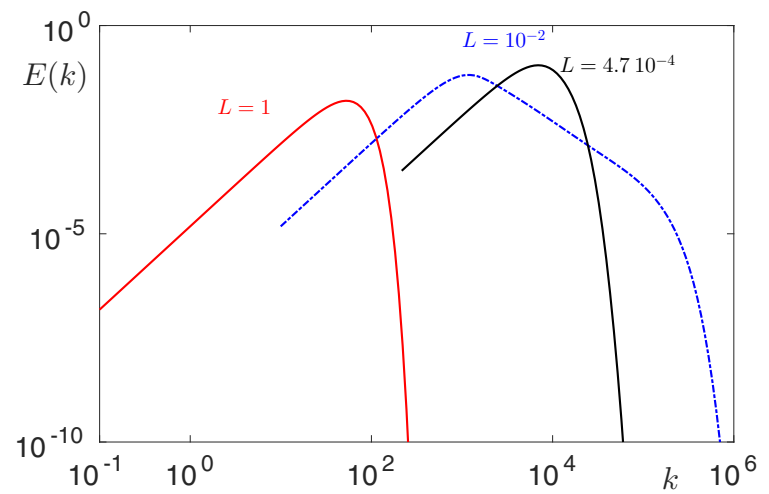

The Reynolds number decrease observed in Fig. 6 (top) is mainly due to the growth of viscosity, while initial conditions have relatively low influence except during the viscous phase. The compression number $\mathrm{Cp}$ (Fig. 6, bottom) increases from its initial low value $\mathrm{Cp}=0.47$ during the $\mathrm{RC}$ phase, and reaches a plateau at about ten times this value during the cascade phase.

The fact that simulations agree well with the scaling laws proposed in Sec. III A is a consequence of the fact that the distribution of energy at large scales remains constant during the compression. This constancy is verified in Fig. 7, for both Saffman and Batchelor turbulences, in which the infrared slopes of turbulent kinetic spectra are maintained over three decades of $k$. In particular, energy backscatter usually alters the slope in Batchelor turbulence, but this seems of little influence here. In addition, the presence of nonlinear transfer over a few decades of scales during the cascade regime is attested by the observed Kolmogorov scaling $k^{-5 / 3}$ in spectra $E(k)$ at $L=10^{-2}$ in Fig. 7. On the contrary, the absence of inertial subrange shows that, during the sudden viscous dissipation phase, energy is systematically dissipated from small to large scales.

In summary, we have evidenced in this section the existence of the self-similar regimes by means of EDQNM simulations at high Reynolds number with long evolution times, thus reaching very small compression parameter $L$. This clearly demonstrates the influence of the initial distribution of energy at large scales, in particular during the viscous dissipation phase. In the following, we show that these results still apply in configurations more relevant to ICF, i.e., when the Reynolds number is smaller and in the presence of inhomogeneities.

\section{Spherical turbulent layer}

An essential question in ICF is how the turbulent ablator or fuel interface eventually contaminates the hot spot at the center of the spherical capsule. We therefore consider here a spherical turbulent layer configuration, more relevant to the ICF problem than homogeneous turbulence. Of course, this case is still simplified for it discards important physical phenomena that are present in ICF, for instance density effects and the stagnation shock in the fuel, which has to be taken into account before the bang time. Nonetheless, it is a progress towards more realistic predictions. Therefore, in this section, we investigate the self-similar solutions and the influence of

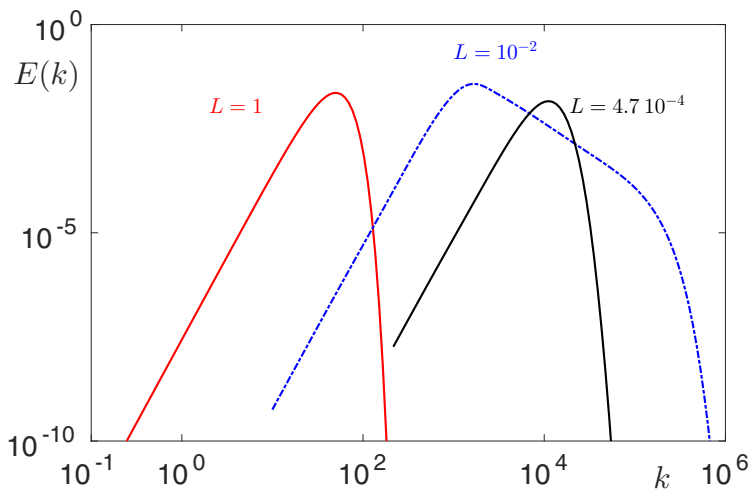

FIG. 7. Kinetic-energy spectra $E(k)$, corresponding to EDQNM simulations of Figs. 5 and 6, taken at different values of the compression parameter $L$. Left: Saffman initial condition $(s=2)$. Right: Batchelor initial condition $(s=4)$. 
large scale perturbations in the case of a spherical turbulent layer under compression using both DNS and EDQNM.

Although some estimates of physical parameters of experimental ICF are very difficult to make, we choose initial conditions that can be relevant to actual flow situations. We evaluate the Reynolds and compression numbers based on the ICF simulations corresponding to the NIF shot N120205 presented in [1]. During the phase considered just before the bang time, the ablator or fuel interface passes from a radius of $340 \mu \mathrm{m}$ to $54 \mu \mathrm{m}$ in $1 \mathrm{~ns}$ giving $\mathcal{S}_{0} \sim 10^{9} \mathrm{~s}^{-1}$. The initial fuel viscosity is around $\nu \sim 10 \mathrm{~cm}^{2} \mathrm{~s}^{-1}$ for $\rho \sim 10 \mathrm{~g} \mathrm{~cm}^{-3}$ and $T \sim 500 \mathrm{eV}$ corresponding to a plasma coupling parameter $\Gamma \sim 0.1$ for the deuterium-tritium fuel. The integral scale and fluctuating velocity are respectively $\ell_{0} \sim 10^{-2} \mathrm{~cm}$ and $u_{0} \sim$ $10^{6} \mathrm{~cm} \mathrm{~s}^{-1}$, leading to $\operatorname{Re}_{0} \sim 10^{3}$ and $\mathrm{Cp}_{0} \sim 10^{-1}$. In our simulations, we therefore choose $\operatorname{Re}_{0}=450$ and $\mathrm{Cp}_{0}=0.1$, figures that are relevant to ICF configurations.

The initial spectra are of von Karman type already introduced in Sec. III B, $E_{0}(k) \sim k^{s} \exp \left[-s / 2\left(k / k_{\text {peak }}\right)^{2}\right]$. These conditions are sufficient to initialize the EDQNM model which assumes homogeneity and isotropy of turbulence. Its predictions will be used to compare to the DNS results. The initialization of the DNS fluctuating velocity field is however more complex, since it requires us to generate an initial spherical layer. In the following the reference frame is still Cartesian, as in the previous DNS. The radial coordinate $r$ with respect to the center of the domain is used, as a convenient way to represent the initial spherical layer and to discuss the the dependency of the spherically averaged statistics.

We therefore proceed as follows:

(1) We generate in spectral space a random incompressible velocity field $\hat{\mathbf{u}}(\mathbf{k})$, as classically done in simulations of HIT.

(2) Using inverse Fourier transform, we obtain the vorticity field $\omega(\mathbf{x})$ in physical space, which is then filtered to $\omega^{F}(\mathbf{x})$ in order to create the spherical layer. The filter is the simple characteristic function of the sphere:

$$
\begin{aligned}
& \omega^{F}(\mathbf{x})=\omega(\mathbf{x}) \text { for } r_{m} \leqslant|\mathbf{x}| \leqslant r_{M}, \text { and } \\
& \omega^{F}(\mathbf{x})=0 \text { elsewhere, }
\end{aligned}
$$

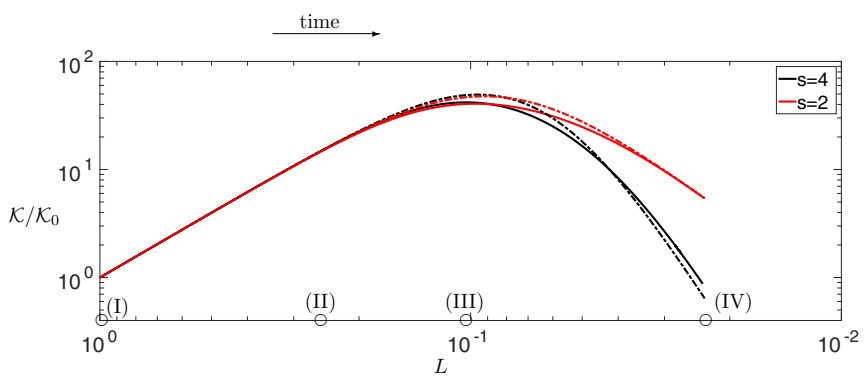

FIG. 8. Evolution of turbulent kinetic energy $\mathcal{K}$ as a function of the compression parameter $L$ for both $1024^{3}$ DNS and EDQNM simulations corresponding to the case of the spherical turbulent layer. Solid line: DNS; dashed line: EDQNM simulation. Saffman and Bachelor initial conditions with respectively $(s=2)$ and $(s=4)$ are used, as indicated in the legend.

where $r_{m}$ and $r_{M}$ define the inner and outer radii of the spherical layer.

(3) The filtered vorticity $\omega^{F}(\mathbf{x})$ is Fourier transformed back to spectral space, and used to generate an incompressible velocity field $\hat{\mathbf{u}}^{F}(\mathbf{k})$ whose amplitudes are adjusted to fit the specified initial spectrum $E_{0}(k)$. Although kinetic energy is mainly concentrated inside the spherical shell, weak irrotational fluctuations of the velocity field appear outside it in order to satisfy incompressibility.

The DNS are done in a three-dimensional $2 \pi$-periodic domain, so that it is important to ensure minimal interaction between adjacent spherical turbulent layers. Therefore, we choose an outer radius $r_{M}$ small enough compared to the size of the domain $H=2 \pi$, so that the velocity decreases sufficiently between the layers. We have experienced that $r_{M} / H=1 / \pi$ is adequate for that, and we also choose an inner radius such that $\left(r_{M}-r_{m}\right) / H=1 /(2 \pi)$. The thickness of the turbulent spherical layer $\ell_{\mathrm{MZ}}$ is determined on the spherically integrated kinetic-energy profiles and corresponds to the region where $\mathcal{K} \geqslant 0.1 \max _{r} \mathcal{K}(r)$. The integral length scale $\ell_{I}$ has to be smaller than $\ell_{\mathrm{MZ}}$ in order to avoid a fast turbulent diffusion
(I)
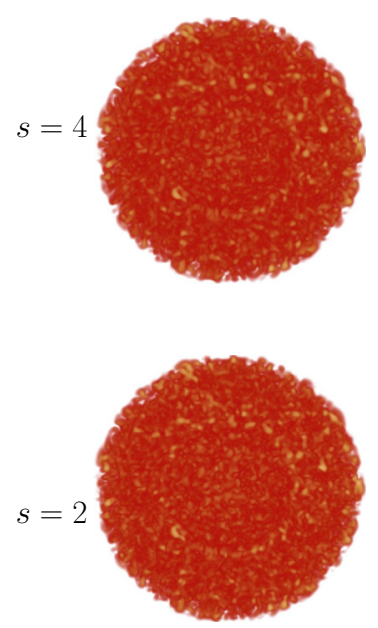

(II)
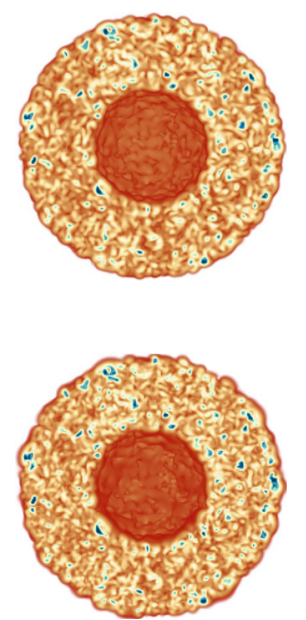

(III)
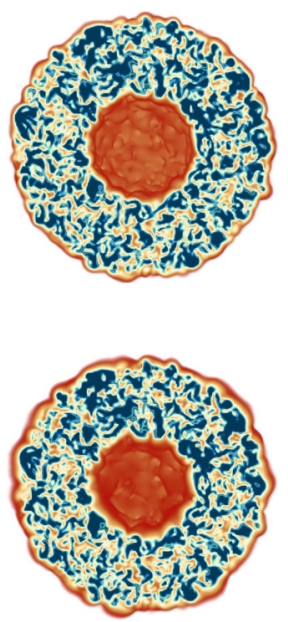

(IV)

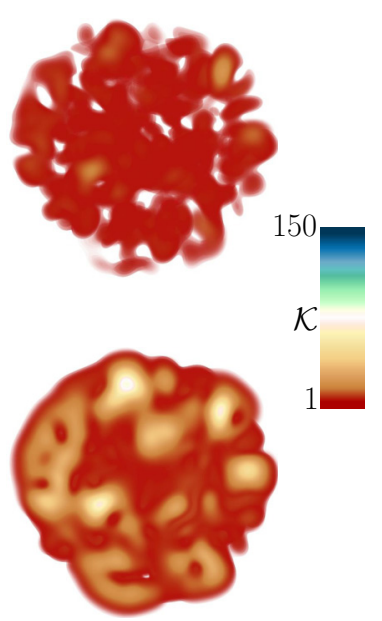

FIG. 9. Distribution in a plane cut of turbulent kinetic energy $\mathcal{K}$ in the DNS at different instants indicated in Fig. 8. Top row: the Batchelor case $(s=4)$; and bottom row: the Saffman case $(s=2)$. The scaling corresponds to that of the moving frame. 

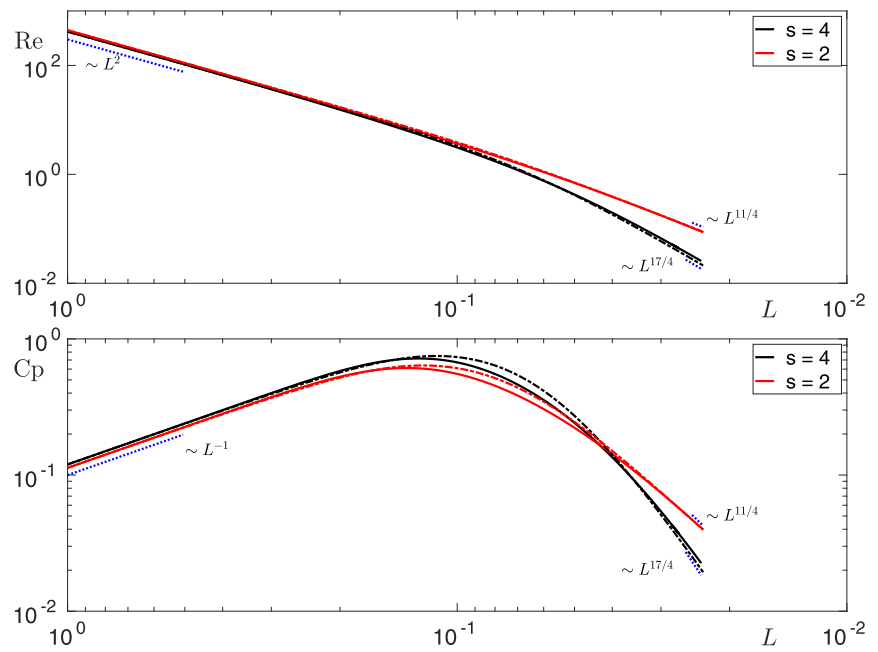

FIG. 10. Evolution of (top) the Reynolds number Re and (bottom) the compression number $\mathrm{Cp}$ as a function of the compression parameter $L$ for the spherical turbulent layer case extracted from DNS (solid line) and EDQNM (dashed line) simulations. Black lines: Batchelor initial condition $(s=4)$. Red lines: Saffman initial condition $(s=2)$.

of the layer during the compression. In consequence, we choose $\ell_{I 0} / \ell_{\mathrm{MZ0}} \sim 0.07$. This scale separation along with the constraint of resolving all the scales of turbulence call for $1024^{3}$ DNS grid points.

Figure 8 shows the evolution of kinetic energy from DNS and EDQNM, and Fig. 9 shows visualizations of the kineticenergy field extracted from DNS at different stages of the compression. The Reynolds and compression numbers Re and $\mathrm{Cp}$, the integral scales $\ell_{I}$, and the turbulent layer size $\ell_{\mathrm{MZ}}$ are plotted in Figs. 10 and 11 respectively. Energy spectra at the same compression stages as the kinetic-energy distribution in Fig. 8 are plotted in Fig. 12. The kinetic energy radial profiles averaged over spherical shells in physical space are shown in Fig. 13.

The kinetic-energy evolution in Fig. 8 exhibits two selfsimilar phases corresponding to rapid compression followed by the viscous dissipation regimes. As expected, the intermediate cascade phase is not observed due to the low value of the initial Reynolds number.

As for the simulations presented previously, DNS and EDQNM agree very well if one considers the nondimensional

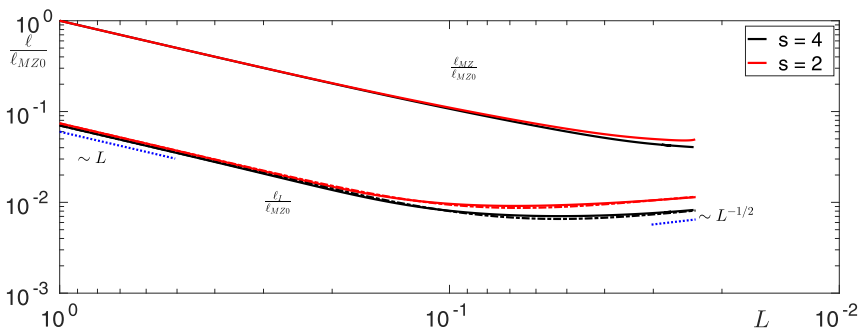

FIG. 11. Evolution of the integral scale $\ell_{I}$ (DNS and EDQNM) and the turbulent layer size $\ell_{\mathrm{MZ}}$ (DNS) as a function of the compression parameter $L$ corresponding to the case of the spherical turbulent layer with (red lines) Saffman and (black lines) Batchelor initial conditions.

parameters (Fig. 10) and the one point statistics $\mathcal{K}, \ell_{I}$ (Fig. 11), but it is also true for the kinetic-energy spectra (Fig. 12). This may appear surprising in the sense that EDQNM is a model for homogeneous isotropic turbulence, whereas the spherical turbulent layer is spatially inhomogeneous. This can be explained by the fact that turbulence length scales remain smaller than the size of the turbulent layer throughout the compression, as shown in Fig. 11. Thus the evolution of turbulent structures is not influenced by the large-scale inhomogeneity, and the flow remains quasihomogeneous as regards its statistics.

The different scaling laws derived in Sec. III A are also recovered in the spherical turbulent layer simulations confirming in particular that viscous dissipation is more important in the Batchelor case compared to the Saffman case, as shown in Fig. 9. One however remarks in Fig. 11 that the integral length scale $\ell_{I}$ is larger in Saffman turbulence than in the Batchelor case. This can be explained by non-negligible nonlinear turbulent transfer around $L_{M}$, since, during the compression phase, $\ell_{I}$ always evolves as $L^{2}$ in the rapid compression regime, while it scales as $L^{-1}$ in the viscous regime.

It is interesting to look at the $\ell_{\mathrm{Mz}}$ 's evolution during the compression in Fig. 11 and at the different kinetic-energy profiles in Fig. 13 which, in the ICF context, would correspond to studying the contamination of the hot spot by the turbulence. At the beginning, for $L \leqslant L_{M}$, the evolution of the turbulent fronts of the turbulent layer is slow and $\ell_{\mathrm{MZ}} \sim L^{2}$. However, the dynamics of $\ell_{\mathrm{MZ}}$ changes when entering the viscous phase which corresponds to a diffusive increase of turbulent kinetic
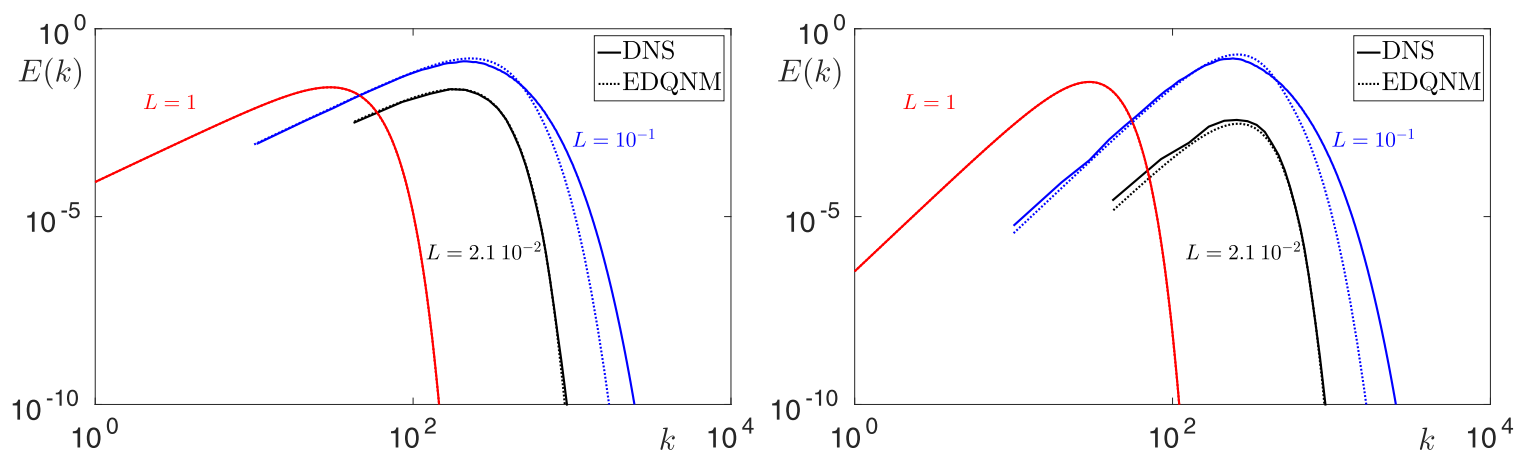

FIG. 12. Energy spectra as a function of wave number $E(k)$ corresponding to the spherical turbulent layer case both in DNS (solid line) and EDQNM (dashed line) and taken at different values of the compression parameter $L$. Right: Batchelor initial condition $(s=4)$. Left: Saffman initial condition $(s=2)$. 

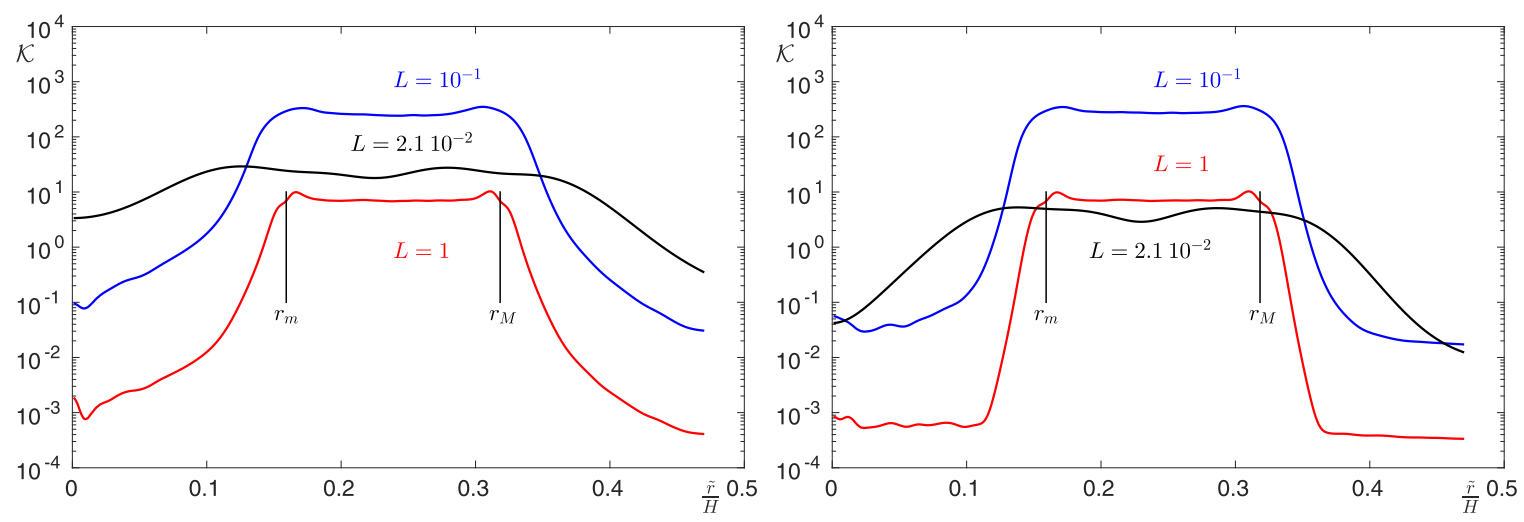

FIG. 13. Spherically integrated kinetic-energy profile extracted from DNS as a function of radius $\tilde{r}$ and taken at different values of the compression parameter $L$. Left: Saffman initial condition $(s=2)$. Right: Batchelor initial condition $(s=4)$.

energy towards the center, as seen in the radial profiles in Fig. 13. This phase also is sensitive to initial conditions since kinetic energy in the center in the Saffman case is higher than in the Batchelor case.

In summary, our simulations show that the sudden viscous phase not only dissipates the turbulent kinetic energy but also enhances its transport, possibly leading in the context of ICF to the contamination of the hot spot. Accordingly, it seems particularly important to predict the time at which the viscous phase occurs, especially if it is before the bang time. From data of the simulations of [1], one can obtain a quantitative estimate of the order of the corresponding time scales. Although some physical phenomena are overlooked and there remain uncertainties concerning their relative magnitude, it seems that the bang time appears before the viscous phase, hence possibly explaining why the contamination of the hot spot is not very important (by bang time the fuel-ablator interface has converged from $340 \mu \mathrm{m}$ to $54 \mu \mathrm{m}$ giving $L_{\text {bang }} \sim 0.16>$ $L_{M}$ ). However, it seems possible to consider different initial perturbations in which the viscous phase may appear earlier than the bang time. This would clearly result in important modifications of the turbulent mixing in the fuel.

\section{CONCLUSION}

In this paper, we study compressed turbulence in a weakly coupled plasma by identifying the different regimes resulting from the competition between turbulence production, nonlinear energy transfer, and viscous dissipation. Depending on the relative importance of these terms, three self-similar regimes can appear, namely rapid compression, cascade, and viscous phases. All three are clearly observed in our DNS and EDQNM simulations. While the rapid compression and viscous phases have been discussed in [6], the cascade phase is precisely characterized thanks to the EDQNM model that permits us to explore very high Reynolds number configurations. In addition, this model has proven its efficiency at lower Reynolds numbers, by matching DNS results regarding one-point statistics and two-point correlation spectra.

For sufficiently large initial Reynolds number and small initial compression number, weakly coupled plasmas under compression experience a growth of kinetic energy due to a rapid compression or cascade phase. However, the viscous phase always prevails at the end of the compression leading to the sudden dissipation phenomenon. By performing a parametric study with the EDQNM model, we have been able to explore the phase space of nondimensional numbers, Re and $\mathrm{Cp}$, and to locate the values of the critical compression parameter at the maximum of kinetic energy corresponding to the beginning of the viscous phase. This also reveals the complex interplay between turbulence production, transfer, and dissipation, leading for some configurations to two successive growth or decay phases of kinetic energy in place of one during the compression.

The scaling laws for the different self-similar regimes have been derived from a theoretical analysis and recovered in DNS and EDQNM simulations. As in other classical turbulence problems, it demonstrates the dependence of the flow dynamics on the initial distribution of energy at large scales. This appears particularly crucial during the viscous phase since it can change drastically the decay exponents of kinetic energy. Consequently, a much higher amount of turbulent fluctuations remain at the end of compression when initial kinetic energy is concentrated at large scales.

Thanks to these results about the refined stages of the flow dynamics and the conditions of their appearance, we were able to extend our study to the case of an inhomogeneous spherical turbulent layer under compression, using parameters representative of ICF capsules. While recovering the different phases already identified in the homogeneous configurations, the simulations permit us to observe the diffusion of the layer towards the center, mimicking the contamination of the hot spot by turbulence. The results show an enhanced enlargement of the layer during the viscous phase, along with a great sensitivity to initial conditions. Therefore, it raises the question of whether the sudden viscous phenomenon is favorable to achieve ignition in ICF. On the one hand, viscous dissipation participates to a global temperature increase by converting kinetic energy into internal energy, increasing the fusion reactions. On the other hand, turbulent mixing can be transported into the core, cooling the hot spot. In all cases, the appearance of the viscous phase indicates an important change in the plasma dynamics and it seems important to figure out when this happens, before or after the bang time for some configurations. 


\section{ACKNOWLEDGMENTS}

We thank Dr. J. Griffond for his help with the spectral code. We are also very grateful to Dr. P. Arnault, Dr. J. Clérouin and Dr. R. Riquier for many discussions concerning transport coefficients and magnetic effects in weakly coupled plasmas. DNS and EDQNM simulations were performed at the French computing center TGCC.
[1] C. R. Weber, D. S. Clark, A. W. Cook, L. E. Busby, and H. F Robey, Inhibition of turbulence in inertial-confinement-fusion hot spots by viscous dissipation, Phys. Rev. E 89, 053106 (2014).

[2] S. I. Braginskii, Transport processes in a plasma, in Reviews of Plasma Physics, edited by M. A. Leontovich (Consultants Bureau, New York, 1965), Vol. I, p. 205.

[3] B. M. Haines, E. L. Vold, K. Molvig, C. Aldrich, and R. Rauenzahn, The effects of plasma diffusion and viscosity on turbulent instability growth, Phys. Plasmas 21, 092306 (2014).

[4] E. L. Vold, A. S. Joglekar, M. I. Ortega, R. Moll, D. Fenn, and K. Molvig, Plasma viscosity with mass transport in spherical inertial confinement fusion implosion simulations, Phys. Plasmas 22, 112708 (2015).

[5] V. Rana, H. Lim, J. Melvin, J. Glimm, B. Cheng, and D. H. Sharp, Mixing with applications to inertial-confinement-fusion implosions, Phys. Rev. E 95, 013203 (2017).

[6] S. Davidovits and N. J. Fisch, Sudden Viscous Dissipation of Compressing Turbulence, Phys. Rev. Lett. 116, 105004 (2016).

[7] D. S. Clark, M. M. Marinak, C. R. Weber, D. C. Eder, S. W. Haan, B. A. Hammel, D. E. Hinkel, O. S. Jones, J. L. Milovich, P. K. Patel, H. F. Robey, J. D. Salmonson, S. M. Sepke, and C. A. Thomas, Radiation hydrodynamics modeling of the highest compression inertial confinement fusion ignition experiment from the national ignition campaign, Phys. Plasmas 22, 022703 (2015).

[8] T. Nishitani and K. Ishii, Similarity transformations of the Navier-Stokes equation, J. Phys. Soc. Jpn. 54, 5461 (1985).

[9] C. Cambon, G. N. Coleman, and N. N. Mansour, Rapid distortion analysis and direct simulation of compressible homogeneous turbulence at finite Mach number, J. Fluid Mech. 257, 641 (1993).

[10] C. Cambon, Y. Mao, and D. Jeandel, On the application of time dependent scaling to the modeling of turbulence undergoing compression, Eur. J. Mech. B-Fluids 11, 683 (1992).

[11] G. K. Batchelor, The role of big eddies in homogeneous turbulence, Proc. R. Soc. London, Ser. A 195, 513 (1949).

[12] G. K. Batchelor, The Theory of Homogeneous Turbulence (Cambridge University Press, Cambridge, England, 1953).
[13] W. K. George, The decay of homogeneous isotropic turbulence, Phys. Fluids A: Fluid Dyn. 4, 1492 (1992).

[14] M. Lesieur, Turbulence in Fluids (Springer, New York, 2008).

[15] S. A. Orszag, Lectures on the statistical theory of turbulence, in Les Houches Summer School 1973 (1977), pp. 273-374.

[16] M. Lesieur and S. Ossia, 3D isotropic turbulence at very high Reynolds numbers: EDQNM study, J. Turb. 1, 007 (2000).

[17] M. Meldi and P. Sagaut, On non-self-similar regimes in homogeneous isotropic turbulence decay, J. Fluid Mech. 711, 364 (2012).

[18] V. Mons, J.-C. Chassaing, T. Gomez, and P. Sagaut, Is isotropic turbulence decay governed by asymptotic behavior of large scales? An eddy-damped quasi-normal Markovian-based data assimilation study, Phys. Fluids 26, 115105 (2014).

[19] A. Burlot, B. J. Gréa, F. S. Godeferd, C. Cambon, and O. Soulard, Large Reynolds number self-similar states of unstably stratified homogeneous turbulence, Phys. Fluids 27, 065114 (2015).

[20] O. Soulard, J. Griffond, and B.-J. Gréa, Large-scale analysis of self-similar unstably stratified homogeneous turbulence, Phys. Fluids 26, 015110 (2014).

[21] O. Soulard, J. Griffond, and B. J. Gréa, Large-scale analysis of unconfined self-similar Rayleigh-Taylor turbulence, Phys. Fluids 27, 095103 (2015).

[22] C. A. Walsh, J. P. Chittenden, K. McGlinchey, N. P. L. Niasse, and B. D. Appelbe, Self-Generated Magnetic Fields in the Stagnation Phase of Indirect-Drive Implosions on the National Ignition Facility, Phys. Rev. Lett. 118, 155001 (2017).

[23] P. Sagaut and C. Cambon, Homogeneous Turbulence Dynamics (Cambridge University Press, Cambridge, 2008), Vol. 10.

[24] O. Soulard, J. Griffond, and D. Souffland, Pseudocompressible approximation and statistical turbulence modeling: Application to shock tube flows, Phys. Rev. E 85, 026307 (2012).

[25] R. S. Rogallo, Numerical experiments in homogeneous turbulence, Technical Report No. 81315. NASA, memo, 1981.

[26] B.-J. Gréa, J. Griffond, and A. Burlot, The effects of variable viscosity on the decay of homogeneous isotropic turbulence, Phys. Fluids 26, 035104 (2014).

[27] G. Viciconte, B.-J. Gréa, and F. S. Godeferd, A spectral model for sudden dissipation effect in turbulent plasma under compression, Congrès Français de Mecanique, Lille, France (2017). 